\title{
Acute pulmonary artery thromboembolism treated with thrombolysis: diagnostic and monitoring uses of transoesophageal echocardiography
}

\author{
Dylmitr Rittoo, George R Sutherland
}

\begin{abstract}
The central pulmonary arteries and any thrombus within them can be imaged by transoesophageal echocardiography. Its use is reported in the diagnosis and subsequent management of a patient with acute pulmonary embolism, whose response to thrombolytic treatment was monitored by further transoesophageal studies.

Transoesophageal echocardiography may be a valuable primary diagnostic procedure in patients who seem to have sustained a significant pulmonary embolus and require urgent intervention.
\end{abstract}

(Br Heart $\mathcal{F} 1993 ; 69: 457-459)$

Pulmonary embolism is a common condition, that has a high mortality if untreated. ${ }^{1}$ Its diagnosis is often a clinical challenge because of its variable manifestations and also because non-invasive techniques cannot reliably establish or exclude the diagnosis in most patients suspected of pulmonary embolism. ${ }^{2}$ A direct view of the structure of and flow within the central pulmonary arteries is now possible by transoesophageal echocardiography (TOE). ${ }^{3}$ We report the diagnostic and monitoring uses of biplane TOE in a patient with acute submassive pulmonary embolism treated with thrombolysis.

\section{Case report}

A 49 year old man was admitted as an emergency with acute shortness of breath. He had been completely well until four months before when mild breathlessness was first noticed on exertion. He was a non-smoker and was not taking any medication. On examination he was breathless at rest and centrally cyanosed. He was in sinus rhythm with a pulse rate of 110 beats/minute and blood pressure of $110 / 80 \mathrm{~mm} \mathrm{Hg}$. His jugular venous pulse was raised. A systolic murmur was heard at the left sternal edge. There was no clinical evidence of deep vein thrombosis in his legs. Chest $x$ ray film showed no abnormality. His electrocardiogram showed an $S_{1}$ $Q_{3} T_{3}$ pattern and $T$ wave inversion in the precordial leads. Arterial $\mathrm{PO}_{2}$ was $8.6 \mathrm{kPa}$ and $\mathrm{PCO}_{2} 4.4 \mathrm{kPa}$. Transthoracic echocardiography showed dilatation of the right ventricle and atrium, flattening of the interventricular septum, mild tricuspid regurgitation on colour flow mapping, and increased pul- monary artery systolic pressure (peak of 70 $\mathrm{mm} \mathrm{Hg}$, as estimated by continuous wave Doppler). Biplane TOE was performed (fig 1). This showed mural thrombus in the right main pulmonary artery. It seemed soft with a central clear area, suggesting a fresh thrombus. Pulmonary angiography performed shortly afterwards showed multiple thrombi in both pulmonary arteries but these seemed to be distal to the main proximal vessels (fig 2A). Thrombus was also confirmed to be present in the left popliteal vein on venography.

Thrombolytic treatment was given, consisting of an intravenous bolus dose of 600000 units of streptokinase, followed by an infusion of 100000 units an hour for 72 hours. This resulted in almost complete resolution of thrombi, as shown by repeat TOE, and confirmed by pulmonary angiography (fig 2B). The patient made an excellent recovery.

\section{Discussion}

Transoesophageal echocardiography has been one of the most important advances in cardiac imaging. The closeness of the probe to the posterior cardiac structures and the use of high frequency transducers allow high resolution imaging. Imaging from the oesophagus also provides real time views of the heart that cannot be obtained by other standard imaging techniques. Current indications for TOE either in the outpatient clinic or in the operating room are numerous and still expanding. ${ }^{3}$ Its role, however, in the assessment of patients suspected of pulmonary embolism is not well documented. Only a few studies have been published, all of which have used single plane TOE.4-6

We used a biplane probe that allows scanning in two orthogonal planes, transverse and longitudinal. With single (transverse) plane TOE, the main pulmonary artery (pulmonary trunk), the entire right pulmonary artery up to its division into major lobar branches, and part of the left pulmonary artery can be imaged. A second (longitudinal) plane has an enhanced view of the main pulmonary artery and the distal segments of the left and right main arteries. Hence thrombus within these central vessels should be readily detected. Thus the diagnosis could be made promptly and treatment started without delay.

Transthoracic echocardiography may provide useful indirect evidence of pulmonary embolism, ${ }^{7}$ such as dilatation of the right ventricle, tricupid valve regurgitation, and increase of pulmonary artery pressure that 

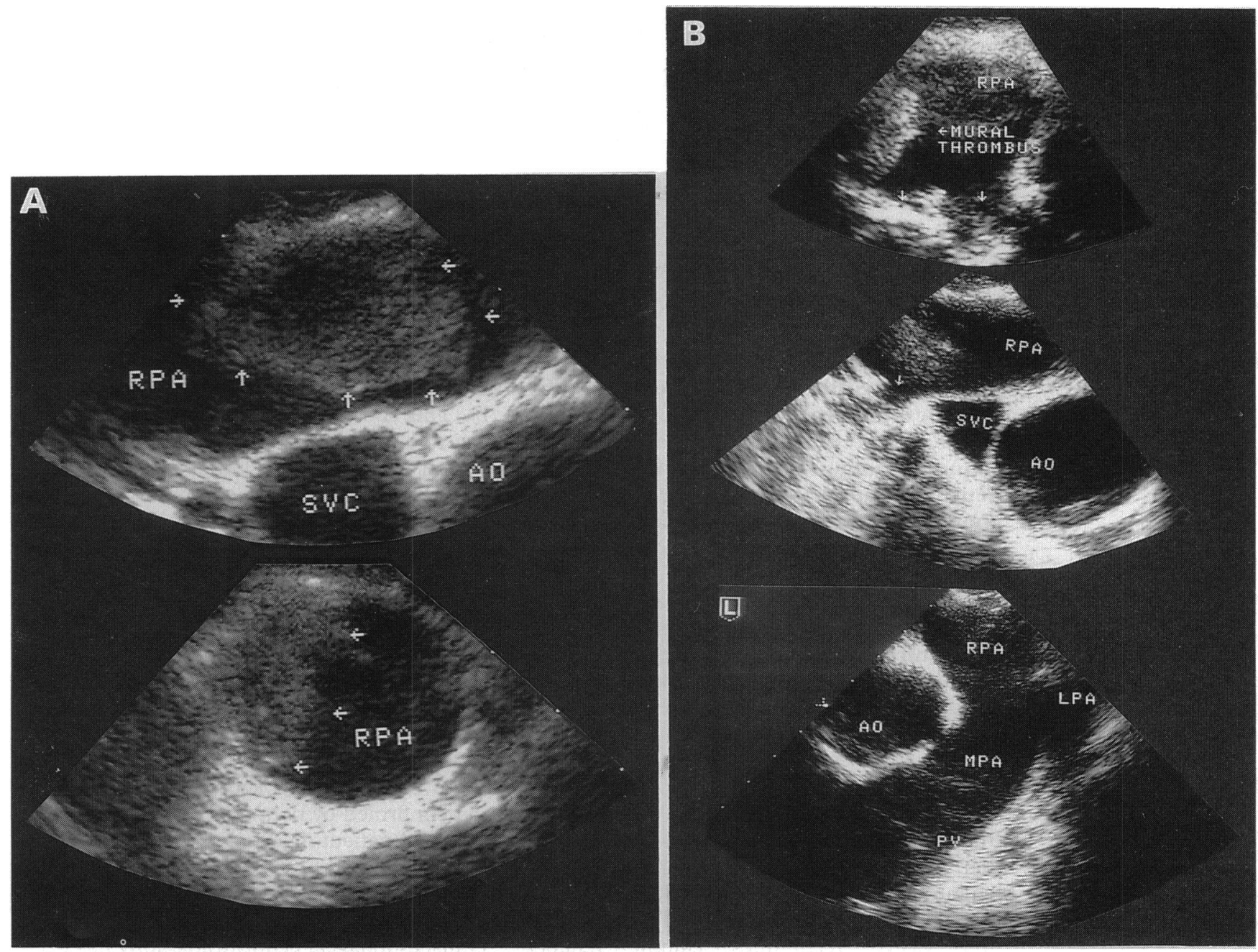

Figure 1 Biplane transoesophageal imaging of the right pulmonary artery. (A) Transverse plane (above) and longitudinal plane (below). Arrows indicate intraluminal thrombus. Note that the size of thrombus can be overestimated by transverse plane scanning. (B) After thrombolysis. Top and middle panels: transverse scan of the distal right pulmonary artery. There is some residual mural thrombus. Vertical arrows show the origin of the lobar arteries. There is white noise artefact in the middle panel.Bottom panel: longitudinal scan of the main pulmonary artery and the proximal right and left branches. Ao, aorta; $L P A$, left pulmonary artery; $M P A$, main pulmonary artery; $P V$, pulmonary valve; RPA, right pulmonary artery; $S V C$, superior vena cava.

can be accurately assessed by continuous wave Doppler. But these changes would only be apparent in patients without previous cardiopulmonary disease when more than $25 \%$ of the pulmonary vascular bed is obstructed. ${ }^{8}$ Occasionally, thrombus may be visible in the

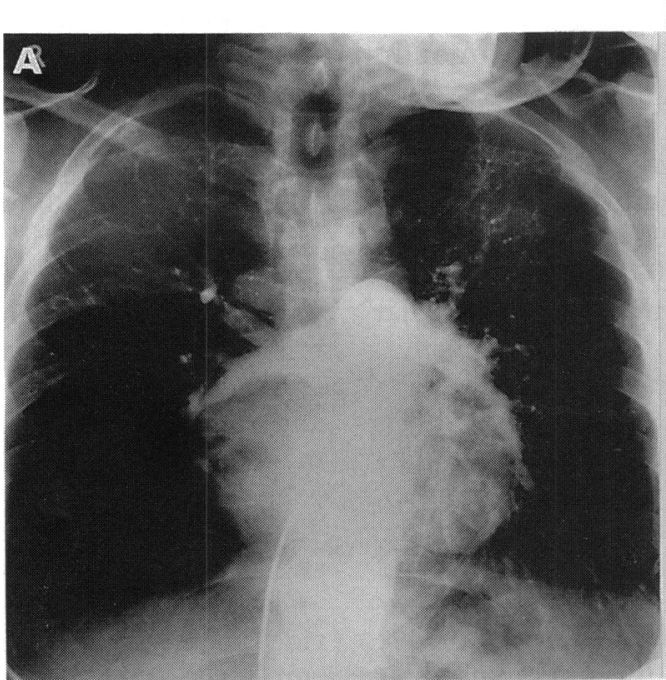

right atrium or ventricle. There is currently growing interest in the use of thrombolytic treatment for pulmonary embolism. Several studies have shown the efficacy of this treatment. ${ }^{8}$ One major drawback has been the need for pulmonary angiography, a facility

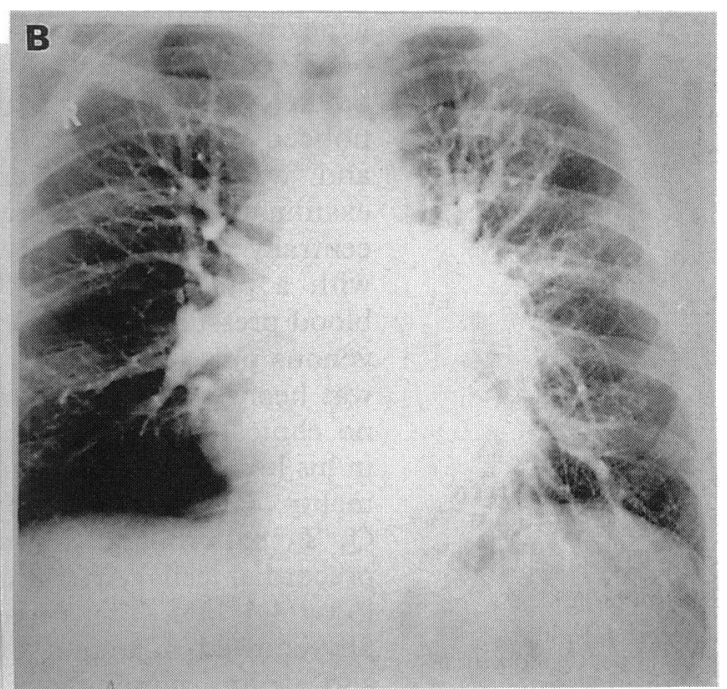

Figure 2 Pulmonary angiograms showing (A) multiple emboli bilaterally and poor peripheral perfusion, and (B) after thrombolytic treatment there is almost complete resolution of thrombus and restoration of blood flow. 
that is not widely available, before initiating thrombolysis. Our case suggests that thrombolysis could be started safely on the basis of TOE findings. A further advantage of TOE over angiography, is that it provides a direct view of thrombus. Fresh thrombus may be distinguished from old organised ones, and resolution during or after thrombolysis can be easily monitored. Another potentially useful role for TOE would be in patients with massive pulmonary emboli in whom it might be possible to perform mechanical clot fragmentation with TOE guidance. ${ }^{910}$

TOE should be considered in patients who clinically seem to have sustained a significant pulmonary embolus and require urgent intervention. Its role, however, is limited to the central pulmonary arteries.

We thank Dr H C Miller for permission to report on this patient.

1 Barrit DW, Jordan SC. Anticoagulant drugs in the treatment of pulmonary embolism. Lancet 1960;i:1309-12.
2 The PIOPED Investigators. Value of the ventilation/perfusion scan in acute pulmonary embolism. Results of the
prospective investigation of pulmonary embolism diagprospective investigation of pulmonary emb
nosis (PIOPED). $A A M A$ 1990;263:2753-9.

3 Seward JB, Khandheria BK, Edwards WD, Oh JK, Freeman WK, Tajik AJ. Biplanar transesophageal echocardiography: anatomic correlations, image orientation, and clinical applications. Mayo Clin Proc 1990;65:1193-213.

4 Nixdorff U, Erbel R, Drexler M, Meyers J. Detection of thromboembolus of the right pulmonary artery by transCardiol 1988;61:488-9.

5 Wittlich N, Erbel R, Todt M, Mohr-Kanaly S, Drexler M, Meyer J. Detection of pulmonary artery thrombi by
transesophageal echocardiography in patients with sustransesophageal echocardiography in patients with suspected pulmonary em

6 Richaud M, Drobinski G, Montalescot G, Salloum J, Bizec JL, Isnard R. Diagnosis of pulmonary embolism by transoesophageal echocardiography. Eur Heart $\mathcal{f}$ 992;13:1000-1.

7 Come PC. Echocardiographic recognition of pulmonary arterial disease and determination of its cause. $A m \mathcal{F}$ Med 1988;84:384-94.

8 Goldhaber SZ. Thrombolysis for pulmonary embolism. Prog Cardiovasc Dis 1991;34:113-34.

9 Brady AJB, Crake T, Oakley CM. Percutaneous catheter fragmentation and distal dispersion of proximal pulmonary embolus. Lancet 1991;338:1186-9.

10 Essop MR, Middlemost S, Skoularis J, Sareli P. Simultaneous mechanical clot fragmentation and pharmacologic thrombolysis in acute pulmonary embolism. Am $\mathcal{F}$ Cardiol 1992;69:427-30. 